\title{
Evaluation of Some Peanut Genotypes under Two Planting Methods and Different Fertilization Levels
}

\author{
El- Far, I.A. ${ }^{1}$; E.A. Ali ${ }^{1}$; W.A. El-Sawy ${ }^{2}$ and A.H. Mohamed ${ }^{2}$ \\ ${ }^{1}$ Agronomy Dept. Agric. Fac. Assiut Univ., Egypt \\ ${ }^{2}$ Oil Crop Res. Dept., Field Crop Res.. Inst. Agric. Res. Center., Giza, Egypt \\ Received on: 8/8/2016 \\ Accepted for publication on: 21/8/2016
}

\begin{abstract}
A field experiment was carried out at Arab Elawamr Research Station Farm, Oil Crops Department, Assiut Governorate, Agriculture Research Center (ARC), Egypt, during the summer of 2013 and 2014 seasons. This work aimd to study the evaluation of some peanut genotypes under two planting methods and different fertilization levels cultivated in sandy calcareous soil. The experiment was laid out in randomized complete block design (RCBD) using split-split-plot arrangement with three replicates. The two planting methods (Farming on the furrows $60 \mathrm{~cm}$ apart and Farming on the terraces each terrace contain two rows $60 \mathrm{~cm}$ apart) were assigned to the main plots. The sub - plots were allocated to three peanut genotypes (Giza 6, Sohag 104 and Sohag 110), while the three fertilization combination ( $20 \mathrm{~m}^{3}$ organic/ fed, $10 \mathrm{~m}^{3}$ organic / fed with $50 \%$ of the recommended NPK and $100 \%$ recommended NPK ) were placed on sub-subplots. The results show that the studied planting methods had a significant effect on most studied traits. Here too, studied genotypes varied significantly in all studied traits except seed and oil yields in the second season. Moreover, all studied traits reacted significantly to studied fertilization levels in the two growing seasons. Also, cultivated Giza 6 genotype by terraces method (M2) received F2 fertilization treatment gained the highest mean values (640.0 and $\left.313.7 \mathrm{~kg} \mathrm{fed}^{-1}\right)$ of seed and oil yields, respectively, in the first season while in the second season the highest mean values ( 818.0 and $396.7 \mathrm{~kg} \mathrm{fed}^{-1}$ ) for seed and oil yields, respectively, were obtained from cultivated Sohage 110 genotype using furrow(M1) planting methods subjected to F2 fertilization treatment.
\end{abstract}

Keywords: Peanut, Genotypes, Planting methods, Cheep manure, seed and oil yields

\section{Introduction}

Peanut (Arachis hypogaea L.) is one of the most important summer legumes in Egypt which thrive in the newly reclaimed sandy soils, more than half of the national production is consumed directly without oil extraction and remaining exported, because its high price in the international commodity market. It's cultivated successfully in newly reclaimed sandy soils which commonly suffers from deficiency or un- availability of most the micronutrients. The peanut seed contains about $25 \%$ to $30 \%$ digestible protein, 45 to $50 \%$ oil, $20 \%$ carbohydrate and $5 \%$ fiber and ash which make a substantial contribution to human nutrition (Ahmad and Rahim, 2007). The cultivated area of peanut in Egypt during 2014 season was about 64000 ha with the total yield production of 205000 ton (FAO, 2014). So, it is of great importance to improve peanut production, by horizontal expansion 
in desert area or by several agricultural practices such as using new genotypes, sowing methods and organo-mineral fertilizers. Many investigators found significant variation between peanut genotypes in growth, yield, yield components and quality due to the differences in genetic structure and their interaction with environmental condition prevailing during growing season (Meena et al., 2014; Mahrous et al., 2015 and Sarkees, 2015). The newly reclaimed sandy soils are poor in organic matter and nutrient elements. Organic matter is a key component of soils affecting their physical, chemical and biological properties and is important as a source of energy and nutrient elements for soil ecosystem. Maintenance of sufficient levels of organic matter in soils is prerequisite for sustainable and high production of crops (Radwan and Awad, 2002). To improve the organic contents of soils for growing crops there are some applications such as planting rotation, various plough techniques, green fertilizer application and animal fertilizer application. The continuous use of high level of chemical fertilizers has led to problem of soil degradation, which is proving detrimental to crop production in our country. So we need balanced fertilization for crop production. Hence combined application of manure may reduce the need for chemical fertilizer, allowing the small farmers to save a part of the cost of production. In addition, global environment pollution can be controlled considerably by reducing the use of chemical fertilizer and increasing the use of manures. One of the used organic- mineral fertilizers is cheep manure which contains many elements which improve the soil fertility and increase the availability of nutrients and consequently increase plant growth and yield. Application of organic fertilizers is one of important practical measures to improve soil fertility. In addition, to providing necessary nutrients for crops and improving soil physico-chemical properties, organic fertilizer is able to enhance soil microbial activity, such as improving activity of soil enzymes and increasing soil microbial biomass (Sun, 2003). Many investigators indicated that the application of organic fertilizer increased the nutrient contents in the soil, their uptake and consequently increased the productivity of crops (Ali and Mahmoud, 2012).

Several concepts have been advanced to explain the improvement of nutrient availability as a result of the application of organic manure due to the reduction in soil $\mathrm{pH}$ (Abd El-Moez et al., 1999) or through the improvement of physical and chemical properties of the treated soils (Orlando et al., 1991; Singh et al., 1991 and Sikora and Azard, 1993). The importance of bio-organic to peanut plants was emphasized by Ahmed et al. (1997) they stated that the highest dry matter accumulation, kernels yield and oil percentage in kernel of peanut were achieved by fertilization with $60 \mathrm{~m}^{3} /$ fed farmyard manure. So, the objectives of this investigation was to evaluation of some peanut genotypes under two planting methods and different fertilization levels.

\section{Materials and Methods}


A field experiment was carried out at Arab Elawamr Research Station Farm, Oil Crops Department, Assiut Governorate, Agriculture Research Center (ARC), Egypt, during the summer of 2013 and 2014 seasons to study the evaluation of some peanut genotypes under two planting methods and different fertilization levels cultivated in sandy calcareous soil. Soil samples were collected to depth of $30 \mathrm{~cm}$ from the experimental sites. Detailed results at the mechanical and chemical analysis of the soil in both seasons are presented in Table 1. The soil texture was sandy.

Table 1. Some physical and chemical properties of experimental site.

\begin{tabular}{|c|c|c|}
\hline Properties & $\mathbf{2 0 1 3}$ & $\mathbf{2 0 1 4}$ \\
\hline Mechanical analysis: & & \\
\hline Sand \% & 85.40 & 87.20 \\
\hline Silt \% & 8.70 & 7.20 \\
\hline Clay \% & 5.90 & 5.60 \\
\hline Soil type & Sandy & Sand \\
\hline Chemical analysis: & & \\
\hline pH 1:1 & 8.21 & 8.43 \\
\hline E C 1:1 & 0.59 & 0.77 \\
\hline Total nitrogen \% & & \\
\hline Phosphorus \% & 0.06 & 0.04 \\
\hline Potassium & 5.14 & 4.88 \\
\hline Total CaCO $\%$ & 0.14 & 0.12 \\
\hline & 27.33 & 32.15 \\
\hline
\end{tabular}

The experiment was laid out in randomized complete block design (RCBD) using split-split-plot arrangement with three replicates. The two planting methods (Farming on the furrows $60 \mathrm{~cm}$ apart and Farming on the terraces each terrace contain two rows $60 \mathrm{~cm}$ apart) were assigned to the main plots. The sub - plots were allocated to three peanut genotypes (Giza 6, Sohag 104 and Sohag 110), while the three fertilization combination $\left(20 \mathrm{~m}^{3}\right.$ organic / fed, $10 \mathrm{~m}^{3}$ organic / fed with $50 \%$ of the recommended NPK and 100\% recommended NPK ) were placed on sub-sub-plots. The organic fertilizer was cheep manure, which contain about 30.7\% organic matter, $0.9 \%$ nitrogen, $0.5 \% \mathrm{P}_{2} \mathrm{O}_{5}$ and $0.8 \% \mathrm{~K}_{2} \mathrm{O}$. The recommended NPK dose of mineral fertilizers was 60,30 and $24 \mathrm{~kg} \mathrm{fed}^{-1}$ for $\mathrm{N}, \mathrm{P}$ and $\mathrm{K}$, respectively. The NPK fertilizers rates were applied on the form of Ammonium nitrate $(33.5 \% \mathrm{~N})$, Calcium super phosphate $\left(15.5 \% \mathrm{P}_{2} \mathrm{O}_{5}\right)$ and Potassium sulfate $\left(48 \% \mathrm{~K}_{2} \mathrm{O}\right)$ as a source of nitrogen, phosphorus and potassium, respectively. Cheep manure and Calcium super phosphate were applied to treated plots during soil preparation to cultivated, while nitrogen and potassium fertilizers were added to treated plots at 30 day after planting. Peanut genotypes (Giza 6, Sohag 104 and Sohag 110) were obtained from oil crop research department, field crops agricultural Research Centre, Giza, Egypt. Seeds were treated by rhizobium spp. to increase its ability to fix nitrogen 
before sowing which was on Jun $20^{\text {th }}$ and $25^{\text {th }}$ in 2013 and 2014 seasons, respectively. Seed rate was $45 \mathrm{~kg} / \mathrm{fed}$. The preceding crop was Onion in the first season and wheat in the second season. The plot size was $10.5 \mathrm{~m}^{2}$. Seeds were sown in hills $15 \mathrm{~cm}$ apart and Thinning at 21 day after planting to secure one plant/hill.

At harvest, a sample of five guarded plants were taken randomly from each sub-sub-plot and plant height $(\mathrm{cm})$, number of branches per plant, number of pods per plant, pods weight per plant (g), seeds weight per plant (g) and 100-seed weight were determine. Pods yield was determined per experimental unit then seed yield in $\mathrm{kg} \mathrm{fed}^{-1}$ was calculated. Seed oil content was determined using Soxhlet apparatus and Petroleum ether as solvent according to A.O.A.C. (1995). Oil yield in $\mathrm{kg} \mathrm{fed}^{-1}$ was estimated by the multiplication of oil percentage by seed yield in $\mathrm{kg} \mathrm{fed}^{-1}$. Shelling percentage $\%$ : estimated from seeds from 100 -pods $/ 100$-pods weight $(\mathrm{g}) *$ 100.

All data collected were analyzed with analysis of variance (ANOVA) Procedures using the MSTAT-C Statistical Software Package (Michigan State University, 1983). Differences between means were compared by Rev. LSD at 5\% level of significant (Gomez and Gomez, 1984).

\section{Results and Discussions}

Vegetative traits: Data presented in Tables 2 and 3 shows that there are significant differences between the tested planting methods in plant height and number of branches plant ${ }^{-1}$ in the two growing seasons concerning number of branches plant ${ }^{-1}$ but in the second season only with respect to plant height. The highest mean values of plant height $(21.0$ and $22.2 \mathrm{~cm}$ in the first and second seasons, respectively and number of branches plant ${ }^{-}$ ${ }^{1}(6.7)$ in both seasons were obtained from $M_{1}$ and $M_{2}$, respectively. Here too, the obtained data revel that there are significant differences between the tested peanut genotypes in plant height in the two growing seasons. Sohag 104 genotype gained the highest mean values of plant height in both seasons of study. This is may be due to the genotypic behaviour in combination with the environmental conditions, which may be suitable for Sohag 104 genotype than the rest genotypes. The obtained results are in a good line with those obtained by Meena et al. (2014), Mahrous et al.(2015) and Sarkees (2015). Also, the data focus that the fertilization had a significant influence on the plant height in both seasons and number of branches plant ${ }^{-}$ ${ }^{1}$ in the second season only. the highest mean values of plant height (22.1 and $22.2 \mathrm{~cm}$ in the first and second seasons, respectively were obtained from F1 and F2 in the first and second seasons, respectively. The previous obtained can be ascribed by increasing Cation Exchange Capacity(CEC) of treated soils by cheep manure or by improvement of soil structure which reflected on water movement and decreasing nutrient losses by leaching 
and deep percolation (Ali and Mahmoud, 2012). The Similar stated was obtained by Ahmed and Osman (2003).

Yield components: Exhibited data in Tables 4 to 7 shows that planting methods, genotypes and fertilization levels affected significantly $(p \leq 0.05)$ on number of pods plant ${ }^{-1}$, weight of pods plant ${ }^{-1}$, seed index and seed weight plant $^{-1}$ in the two growing seasons except planting methods in the first seasons concerning number of pods plant ${ }^{-1}$ and seed yield plant ${ }^{-1}$. The highest mean values of pods number plant ${ }^{-1}(35.9)$ pod plant $^{-1}$ in the second season, weight of pods plant ${ }^{-1}(57.5$ and 56.3 in the first and second seasons, respectively), seed index (80.7 and 85.7) in the first and second seasons, respectively and seeds yield plant $^{-1}(36.6)$ in the second season were obtained from $\mathrm{M}_{1}$ (furrow planting) except seed index in the second season which reacted to $\mathrm{M}_{2}$ (terraces planting). Furthermore, the studied genotypes varied significantly in previous traits. Thus, Giza 6 genotype produce the highest mean values of pods number plant ${ }^{-1}$ (36.2 and 35.9) in the first and second seasons, respectively, weight of pods plant ${ }^{-1}(62.8$ in the first season), seed yield plant $^{-1}$ (44.8) in the first season, while Sohag110 genotype produced the highest mean values of seed index $(81.7$ and $86.4 \mathrm{~g})$ in the first and second seasons, respectively. This is may be due to the genotypic behaviour in combination with the environmental conditions. Similar findings were recorded by Meena et al. (2014), Mahrous et al. (2015) and Sarkees (2015). The maximum mean values of pods number plant ${ }^{-1}(31.6$ and 37.6 in the first and second seasons, respectively) and pods weight plant ${ }^{-1}$ (57.9 and $58.5 \mathrm{~g}$ in the first and second seasons, respectively) were obtained from F3 (100\% NPK) and F2 (50\% NPK with $\left.10 \mathrm{~m}^{3}\right)$ cheep manure fed- ${ }^{1}$, respectively. Seed index trait reacted significantly to F3 fertilizer level in the first seasons and produced $83.4 \mathrm{~g}$ per 100 seed being $86.3 \mathrm{~g}$ per 100 seed in the second season under F1 fertilizer level $\left(20 \mathrm{~m}^{3}\right.$ cheep manure without mineral fertilizers). Here too, the application of $50 \%$ of the recommended NPK with $10 \mathrm{~m}^{3}$ cheep manure fed- ${ }^{1}(\mathrm{~F} 2)$ produced the maximum mean values of seed yield $\operatorname{plant}^{-1}(40.2$ and $37.5 \mathrm{~g}$ in the first and second seasons, respectively). The previous finding concern seeds 
Table 2. Effect of planting methods(M), genotypes(G), fertilization(F) and their interactions involved on plant height $(\mathrm{cm})$

\begin{tabular}{|c|c|c|c|c|c|c|c|c|c|}
\hline \multicolumn{2}{|c|}{ Seasons } & \multicolumn{3}{|c|}{2013} & \multirow{2}{*}{ Mean } & \multicolumn{3}{|c|}{2014} & \multirow{2}{*}{ Mean } \\
\hline $\begin{array}{c}\begin{array}{c}\text { Planting } \\
\text { methods(M) }\end{array} \\
\text { mothen }\end{array}$ & $\begin{array}{c}\text { Fertilization } \\
(\mathbf{F})\end{array}$ & G1 & G2 & G3 & & G1 & G2 & G3 & \\
\hline \multirow{3}{*}{ M1 } & F1 & 24.0 & 22.7 & 20.3 & 22.3 & 21.0 & 22.0 & 22.0 & 21.7 \\
\hline & F2 & 21.0 & 23.7 & 20.0 & 21.6 & 23.3 & 26.3 & 21.3 & 23.7 \\
\hline & F3 & 20.3 & 21.7 & 19.3 & 20.4 & 20.0 & 25.0 & 19.0 & 21.3 \\
\hline \multicolumn{2}{|c|}{ Mean } & 21.8 & 22.7 & 19.9 & 21.0 & 21.4 & 24.4 & 20.8 & 22.2 \\
\hline \multirow{3}{*}{ M2 } & F1 & 20.3 & 22.3 & 22.7 & 21.8 & 19.3 & 22.3 & 20.7 & 20.8 \\
\hline & F2 & 20.3 & 20.7 & 19.0 & 20.0 & 20.3 & 23.0 & 18.7 & 20.7 \\
\hline & F3 & 20.7 & 20.7 & 20.7 & 20.7 & 19.7 & 22.0 & 19.3 & 20.3 \\
\hline \multicolumn{2}{|c|}{ Mean } & 20.4 & 21.2 & 20.8 & 20.8 & 19.8 & 22.4 & 19.6 & 20.6 \\
\hline \multicolumn{2}{|c|}{ General mean } & 21.1 & 22.0 & 20.3 & 21.1 & 20.6 & 23.4 & 20.2 & 21.4 \\
\hline \multirow{3}{*}{$\mathbf{G} \times \mathbf{F}$} & F1 & 22.2 & 22.5 & 21.5 & 22.1 & 20.2 & 22.2 & 21.3 & 21.2 \\
\hline & F2 & 20.7 & 22.2 & 19.5 & 20.8 & 21.8 & 24.7 & 20.0 & 22.2 \\
\hline & F3 & 20.5 & 21.2 & 20.0 & 20.6 & 19.8 & 23.5 & 19.2 & 20.8 \\
\hline \multicolumn{2}{|c|}{ F test and L.S.D' } & \multicolumn{2}{|c|}{ F test } & \multicolumn{2}{|c|}{ LSD' $^{\prime}$} & \multicolumn{2}{|c|}{ F test } & \multicolumn{2}{|c|}{ LSD' $^{\prime}$} \\
\hline \multicolumn{2}{|c|}{$\mathbf{M}$} & \multicolumn{2}{|c|}{ NS } & \multicolumn{2}{|c|}{$\begin{array}{l}------ \\
\end{array}$} & \multicolumn{2}{|c|}{$*$} & \multicolumn{2}{|c|}{------- } \\
\hline \multicolumn{2}{|c|}{$\mathbf{G}$} & \multicolumn{2}{|c|}{$*$} & \multicolumn{2}{|c|}{1.28} & \multicolumn{2}{|c|}{ * } & \multicolumn{2}{|c|}{0.75} \\
\hline \multicolumn{2}{|c|}{$\mathbf{F}$} & \multicolumn{2}{|c|}{$*$} & \multicolumn{2}{|c|}{1.19} & \multicolumn{2}{|c|}{ * } & \multicolumn{2}{|c|}{0.72} \\
\hline \multicolumn{2}{|c|}{$\mathbf{M} \times \mathbf{G}$} & \multicolumn{2}{|c|}{$\mathrm{NS}$} & & & & & & \\
\hline & & & & & & & & & \\
\hline & & & & & & & & & \\
\hline $\mathbf{M}>$ & $\mathbf{3} \times \mathbf{F}$ & & & & & & & & \\
\hline
\end{tabular}

NS $=$ Non-significant differences.

$*, * *$ indicated a significant and highly significant at $5 \%$ and $1 \%$ levels of probability.

$\mathrm{LSD}^{\prime}=$ Revised least significant difference.

$\mathrm{M} 1=$ farming on the furrows, M2 = farming in terrace,

G1 = Giza 6, G2 = Sohag 104, G3 = Sohag 110,

$\mathrm{F} 1=20 \mathrm{~m} 3$ organic $\backslash$ fed. , F2 $=10 \mathrm{~m} 3$ organic $\backslash$ fed. with $50 \%$ of recommended NPK and F3

$=100 \%$ of recommended NPK 
Table 3. Effect of planting methods(M), genotypes(G), fertilization(F) and their interactions involved on branches number plant-1

\begin{tabular}{|c|c|c|c|c|c|c|c|c|c|}
\hline \multicolumn{2}{|c|}{ Seasons } & \multicolumn{3}{|c|}{2013} & \multirow{2}{*}{ Mean } & \multicolumn{3}{|c|}{2014} & \multirow{2}{*}{ Mean } \\
\hline $\begin{array}{c}\text { Planting } \\
\text { methods(M) }\end{array}$ & $\begin{array}{c}\text { Fertilization } \\
(\mathbf{F})\end{array}$ & G1 & G2 & G3 & & G1 & G2 & G3 & \\
\hline \multirow{3}{*}{ M1 } & F1 & 5.9 & 5.8 & 6.3 & 6.0 & 6.3 & 6.5 & 6.3 & 6.0 \\
\hline & F2 & 6.4 & 6.6 & 6.8 & 6.6 & 6.9 & 6.7 & 6.2 & 6.6 \\
\hline & F3 & 6.6 & 6.5 & 6.6 & 6.5 & 6.7 & 6.8 & 7.0 & 6.8 \\
\hline \multicolumn{2}{|c|}{ Mean } & 6.3 & 6.3 & 6.6 & 6.4 & 6.6 & 6.4 & 6.5 & 6.5 \\
\hline \multirow{3}{*}{ M2 } & F1 & 7.2 & 6.9 & 6.6 & 6.9 & 7.0 & 7.2 & 6.8 & 7.0 \\
\hline & F2 & 6.8 & 7.0 & 6.3 & 6.7 & 6.2 & 6.9 & 6.1 & 6.4 \\
\hline & F3 & 6.7 & 6.6 & 6.7 & 6.6 & 6.2 & 6.9 & 6.5 & 6.5 \\
\hline \multicolumn{2}{|c|}{ Mean } & 6.9 & 6.8 & 6.5 & 6.7 & 6.5 & 7.0 & 6.5 & 6.7 \\
\hline \multicolumn{2}{|c|}{ General mean } & 6.6 & 6.5 & 6.6 & 6.5 & 6.6 & 6.7 & 6.5 & 6.6 \\
\hline \multirow{3}{*}{$\mathbf{G} \times \mathbf{F}$} & F1 & 6.6 & 6.3 & 6.5 & 6.4 & 6.7 & 6.4 & 6.6 & 6.6 \\
\hline & F2 & 6.6 & 6.8 & 6.6 & 6.7 & 6.6 & 6.8 & 6.2 & 6.5 \\
\hline & F3 & 6.6 & 6.5 & 6.6 & 6.5 & 6.5 & 6.9 & 6.8 & 6.7 \\
\hline \multicolumn{2}{|c|}{ F test and L.S.D' } & \multicolumn{2}{|c|}{ F test } & \multicolumn{2}{|c|}{ LSD $^{\prime}$} & \multicolumn{2}{|c|}{$F$ test } & \multicolumn{2}{|c|}{ LSD $^{\prime}$} \\
\hline \multicolumn{2}{|c|}{$\mathbf{M}$} & \multicolumn{2}{|c|}{$*$} & \multicolumn{2}{|c|}{ |------- } & \multicolumn{2}{|c|}{$*$} & \multicolumn{2}{|c|}{------- } \\
\hline \multicolumn{2}{|c|}{$\mathbf{G}$} & \multicolumn{2}{|c|}{ Ns } & \multicolumn{2}{|c|}{------- } & \multicolumn{2}{|c|}{ NS } & \multicolumn{2}{|c|}{------- } \\
\hline \multicolumn{2}{|c|}{$\mathbf{F}$} & \multicolumn{2}{|c|}{ Ns } & \multicolumn{2}{|c|}{------- } & \multicolumn{2}{|c|}{$*$} & \multicolumn{2}{|c|}{0.13} \\
\hline \multicolumn{2}{|c|}{$\mathbf{M} \times \mathbf{G}$} & \multicolumn{2}{|c|}{$*$} & & & & & & \\
\hline & $\times \mathbf{F}$ & & & & & & & & \\
\hline & & & & & & & & & \\
\hline $\mathbf{M} \times$ & $\mathbf{G} \times \mathbf{F}$ & & & & & & & & \\
\hline
\end{tabular}

Table 4. Effect of planting methods(M), genotypes(G), fertilization(F) and their interactions involved on number of pods plant-1

\begin{tabular}{|c|c|c|c|c|c|c|c|c|c|}
\hline \multicolumn{2}{|c|}{ Seasons } & \multicolumn{3}{|c|}{2013} & \multirow{2}{*}{ Mean } & \multicolumn{3}{|c|}{2014} & \multirow[b]{2}{*}{ Mean } \\
\hline $\begin{array}{c}\text { Planting } \\
\text { methods(M) }\end{array}$ & $\begin{array}{c}\text { Fertilization } \\
\text { (F) }\end{array}$ & G1 & G2 & G3 & & G1 & G2 & G3 & \\
\hline \multirow{3}{*}{ M1 } & F1 & 27.5 & 22.8 & 23.3 & 24.5 & 28.7 & 33.0 & 27.7 & 29.8 \\
\hline & F2 & 44.1 & 31.0 & 33.3 & 36.1 & 42.8 & 43.3 & 41.4 & 42.5 \\
\hline & F3 & 36.6 & 27.9 & 25.8 & 30.1 & 47.3 & 31.2 & 27.6 & 35.4 \\
\hline \multicolumn{2}{|c|}{ Mean } & 36.0 & 27.2 & 27.4 & 30.2 & 39.6 & 35.8 & 32.2 & 35.9 \\
\hline \multirow{3}{*}{ M2 } & F1 & 44.0 & 27.4 & 25.9 & 32.4 & 40.3 & 42.1 & 36.9 & 39.7 \\
\hline & F2 & 21.4 & 27.1 & 28.6 & 25.7 & 29.6 & 33.1 & 35.3 & 32.7 \\
\hline & F3 & 43.8 & 24.9 & 30.8 & 33.2 & 26.4 & 25.8 & 22.2 & 24.8 \\
\hline \multicolumn{2}{|c|}{ Mean } & 36.4 & 26.5 & 28.4 & ---- & 32.1 & 33.6 & 31.5 & ----- \\
\hline \multicolumn{2}{|c|}{ General mean } & 36.2 & 26.9 & 28.0 & 30.3 & 35.9 & 34.7 & 31.9 & 34.1 \\
\hline \multirow{3}{*}{$\mathbf{G} \times \mathbf{F}$} & F1 & 35.8 & 25.1 & 24.6 & 28.5 & 34.5 & 37.5 & 32.3 & 34.8 \\
\hline & F2 & 32.7 & 29.1 & 31.0 & 30.9 & 36.2 & 38.2 & 38.4 & 37.6 \\
\hline & F3 & 40.2 & 26.4 & 28.3 & 31.6 & 36.9 & 28.5 & 24.9 & 30.1 \\
\hline \multicolumn{2}{|c|}{ F test and L.S.D' } & \multicolumn{2}{|c|}{ F test } & \multicolumn{2}{|c|}{ LSD $^{\prime}$} & \multicolumn{2}{|c|}{ F test } & \multicolumn{2}{|c|}{ LSD' $^{\prime}$} \\
\hline \multicolumn{2}{|c|}{$\mathbf{M}$} & \multicolumn{2}{|c|}{ NS } & & \multicolumn{2}{|c|}{$*$} & \\
\hline \multicolumn{2}{|c|}{$\mathbf{G}$} & \multicolumn{2}{|c|}{$*$} & \multicolumn{2}{|c|}{1.28} & \multicolumn{2}{|c|}{$*$} & \multicolumn{2}{|c|}{2.57} \\
\hline \multicolumn{2}{|c|}{$\mathbf{F}$} & \multicolumn{2}{|c|}{$*$} & \multicolumn{2}{|c|}{1.47} & \multicolumn{2}{|c|}{$*$} & \multicolumn{2}{|c|}{2.19} \\
\hline \multicolumn{2}{|c|}{$\mathbf{M} \times \mathbf{G}$} & \multicolumn{2}{|c|}{ NS } & & & & & & \\
\hline & $\times \mathbf{F}$ & & & & & & & & \\
\hline & $\times \mathbf{F}$ & & & & & & & & \\
\hline $\mathbf{M}>$ & $\mathbf{G} \times \mathbf{F}$ & & & & & & & & \\
\hline
\end{tabular}


Table 5. Effect of planting methods(M), genotypes(G), fertilization(F) and their interactions involved on weight of pods plant-1 (gm)

\begin{tabular}{|c|c|c|c|c|c|c|c|c|c|}
\hline \multicolumn{2}{|c|}{ Seasons } & \multicolumn{3}{|c|}{2013} & \multirow{2}{*}{ Mean } & \multicolumn{3}{|c|}{2014} & \multirow{2}{*}{ Mean } \\
\hline $\begin{array}{c}\text { Planting } \\
\text { methods(M) }\end{array}$ & $\begin{array}{l}\text { Fertilization } \\
\text { (F) }\end{array}$ & G1 & G2 & G3 & & G1 & G2 & G3 & \\
\hline \multirow{3}{*}{ M1 } & F1 & 51.3 & 45.0 & 50.0 & 48.8 & 44.0 & 50.0 & 49.7 & 47.9 \\
\hline & F2 & 75.0 & 55.0 & 66.3 & 65.4 & 58.3 & 80.0 & 64.0 & 67.4 \\
\hline & F3 & 65.3 & 55.0 & 54.7 & 58.3 & 61.0 & 59.0 & 41.0 & 53.7 \\
\hline \multicolumn{2}{|c|}{ Mean } & 63.9 & 51.7 & 57.0 & 57.5 & 54.4 & 63.0 & 51.6 & 56.3 \\
\hline \multirow{3}{*}{ M2 } & F1 & 75.7 & 52.0 & 55.7 & 61.1 & 54.0 & 64.7 & 56.7 & 58.4 \\
\hline & F2 & 40.0 & 50.3 & 54.3 & 48.2 & 40.7 & 55.0 & 53.0 & 49.6 \\
\hline & F3 & 69.3 & 43.0 & 60.0 & 57.4 & 37.3 & 38.7 & 40.0 & 38.8 \\
\hline \multicolumn{2}{|c|}{ Mean } & 61.7 & 48.4 & 56.7 & 55.6 & 44.0 & 52.8 & 50.0 & 48.9 \\
\hline \multicolumn{2}{|c|}{ General mean } & 62.8 & 50.1 & 56.8 & 56.5 & 49.2 & 57.9 & 50.8 & 52.6 \\
\hline \multirow{3}{*}{$\mathbf{G} \times \mathbf{F}$} & F1 & 63.5 & 48.5 & 52.8 & 54.9 & 49.0 & 57.3 & 53.2 & 53.2 \\
\hline & F2 & 57.5 & 52.7 & 60.3 & 56.8 & 49.5 & 67.5 & 58.5 & 58.5 \\
\hline & F3 & 67.3 & 49.0 & 57.3 & 57.9 & 49.2 & 48.8 & 40.7 & 46.2 \\
\hline \multicolumn{2}{|c|}{ F test and L.S.D' } & \multicolumn{2}{|c|}{$F$ test } & \multicolumn{2}{|c|}{ LSD $^{\prime}$} & \multicolumn{2}{|c|}{ F test } & \multicolumn{2}{|c|}{ LSD' } \\
\hline \multicolumn{2}{|c|}{$\mathbf{M}$} & \multicolumn{2}{|c|}{$*$} & \multicolumn{2}{|c|}{------- } & \multicolumn{2}{|c|}{$*$} & \multicolumn{2}{|c|}{------- } \\
\hline \multicolumn{2}{|c|}{$\mathbf{G}$} & \multicolumn{2}{|c|}{ * } & \multicolumn{2}{|c|}{1.70} & \multicolumn{2}{|c|}{ * } & \multicolumn{2}{|c|}{3.80} \\
\hline \multicolumn{2}{|c|}{$\mathbf{F}$} & \multicolumn{2}{|c|}{ * } & \multicolumn{2}{|c|}{2.45} & \multicolumn{2}{|c|}{ * } & \multicolumn{2}{|c|}{2.40} \\
\hline \multicolumn{2}{|c|}{$\mathbf{M} \times \mathbf{G}$} & \multicolumn{2}{|c|}{ NS } & & & & & & \\
\hline & $x \mathbf{F}$ & & & & & & & & \\
\hline & $\mathbf{x}$ & & & & & & & & \\
\hline & $\mathbf{G} \times \mathbf{F}$ & & & & & & & & \\
\hline
\end{tabular}

Table 6. Effect of planting methods(M), genotypes(G), fertilization(F) and their interactions involved on seed index (g)

\begin{tabular}{|c|c|c|c|c|c|c|c|c|c|}
\hline \multicolumn{2}{|c|}{ Seasons } & \multicolumn{3}{|c|}{2013} & \multirow{2}{*}{ Mean } & \multicolumn{3}{|c|}{2014} & \multirow{2}{*}{ Mean } \\
\hline $\begin{array}{c}\text { Planting } \\
\text { methods(M) }\end{array}$ & $\begin{array}{c}\text { Fertilization } \\
\text { (F) }\end{array}$ & G1 & G2 & G3 & & G1 & G2 & G3 & \\
\hline \multirow{3}{*}{ M1 } & F1 & 74.3 & 98.7 & 67.0 & 80.0 & 87.2 & 88.1 & 82.6 & 85.9 \\
\hline & F2 & 69.6 & 77.5 & 82.1 & 76.4 & 84.7 & 85.0 & 86.6 & 85.4 \\
\hline & F3 & 80.0 & 75.0 & 79.2 & 78.7 & 82.3 & 86.6 & 87.9 & 85.6 \\
\hline \multicolumn{2}{|c|}{ Mean } & 74.6 & 83.7 & 76.1 & 78.4 & 84.7 & 86.5 & 85.7 & 85.7 \\
\hline \multirow{3}{*}{ M2 } & F1 & 62.6 & 78.9 & 79.0 & 73.5 & 86.8 & 84.8 & 88.5 & 86.7 \\
\hline & F2 & 81.6 & 77.5 & 80.2 & 79.8 & 77.4 & 82.9 & 85.6 & 81.9 \\
\hline & F3 & 90.1 & 73.4 & 102.7 & 88.7 & 80.0 & 80.1 & 87.0 & 82.3 \\
\hline \multicolumn{2}{|c|}{ Mean } & 78.1 & 76.6 & 87.3 & 80.7 & 81.4 & 82.6 & 87.0 & 83.7 \\
\hline \multicolumn{2}{|c|}{ General mean } & 76.3 & 80.1 & 81.7 & 79.4 & 83.1 & 84.5 & 86.4 & 84.7 \\
\hline \multirow{3}{*}{$\mathbf{G} \times \mathbf{F}$} & F1 & 68.4 & 88.8 & 73.0 & 76.7 & 87.0 & 86.4 & 85.5 & 86.3 \\
\hline & F2 & 75.6 & 77.5 & 81.2 & 78.2 & 81.0 & 83.9 & 86.1 & 83.7 \\
\hline & F3 & 85.0 & 74.2 & 91.0 & 83.4 & 81.1 & 83.3 & 87.4 & 84.0 \\
\hline \multicolumn{2}{|c|}{ F test and L.S.D' } & \multicolumn{2}{|c|}{$F$ test } & \multicolumn{2}{|c|}{ LSD $^{\prime}$} & \multicolumn{2}{|c|}{$F$ test } & \multicolumn{2}{|c|}{ LSD $^{\prime}$} \\
\hline \multicolumn{2}{|c|}{$\mathbf{M}$} & \multicolumn{2}{|c|}{$*$} & \multicolumn{2}{|c|}{------- } & \multicolumn{2}{|c|}{$*$} & \multicolumn{2}{|c|}{------- } \\
\hline \multicolumn{2}{|c|}{ G } & \multicolumn{2}{|c|}{$*$} & \multicolumn{2}{|c|}{1.81} & \multicolumn{2}{|c|}{$*$} & \multicolumn{2}{|c|}{1.73} \\
\hline \multicolumn{2}{|c|}{$\mathbf{F}$} & \multicolumn{2}{|c|}{ * } & \multicolumn{2}{|c|}{2.23} & \multicolumn{2}{|c|}{$*$} & \multicolumn{2}{|c|}{0.79} \\
\hline \multicolumn{2}{|c|}{$\mathbf{M} \times \mathbf{G}$} & \multicolumn{2}{|c|}{ * } & & & & & & \\
\hline & & & & & & & & & \\
\hline & & & & & & & & & \\
\hline $\mathbf{M} \times$ & $\mathbf{G} \times \mathbf{F}$ & & & & & & & & \\
\hline
\end{tabular}


Table 7. Effect of planting methods(M), genotypes(G), fertilization(F) and their interactions involved on weight of seeds plant-1 (g)

\begin{tabular}{|c|c|c|c|c|c|c|c|c|c|}
\hline \multicolumn{2}{|c|}{ Seasons } & \multicolumn{3}{|c|}{2013} & \multirow{2}{*}{ Mean } & \multicolumn{3}{|c|}{2014} & \multirow{2}{*}{ Mean } \\
\hline $\begin{array}{c}\text { Planting } \\
\text { methods(M) }\end{array}$ & $\begin{array}{l}\text { Fertilization } \\
\text { (F) }\end{array}$ & G1 & G2 & G3 & & G1 & G2 & G3 & \\
\hline \multirow{3}{*}{ M1 } & F1 & 38.1 & 31.6 & 33.3 & 34.3 & 29.1 & 33.4 & 33.5 & 32.0 \\
\hline & F2 & 55.3 & 36.8 & 44.4 & 45.5 & 40.7 & 52.9 & 40.9 & 44.8 \\
\hline & F3 & 42.7 & 37.7 & 36.8 & 39.1 & 32.7 & 37.8 & 28.2 & 32.9 \\
\hline \multicolumn{2}{|c|}{ Mean } & 45.3 & 35.4 & 38.2 & 39.6 & 34.1 & 41.4 & 34.2 & 36.6 \\
\hline \multirow{3}{*}{ M2 } & F1 & 53.4 & 37.1 & 39.4 & 43.3 & 36.8 & 41.2 & 37.4 & 38.5 \\
\hline & F2 & 29.4 & 36.1 & 39.2 & 34.9 & 25.6 & 37.1 & 27.8 & 30.2 \\
\hline & F3 & 49.9 & 30.7 & 39.7 & 40.1 & 24.2 & 23.5 & 25.9 & 24.6 \\
\hline \multicolumn{2}{|c|}{ Mean } & 44.2 & 34.7 & 39.4 & 39.4 & 28.9 & 34.0 & 30.4 & 31.1 \\
\hline \multicolumn{2}{|c|}{ General mean } & 44.8 & 35.0 & 38.8 & 39.5 & 31.5 & 37.6 & 32.3 & 33.8 \\
\hline \multirow{3}{*}{$\mathbf{G} \times \mathbf{F}$} & F1 & 45.7 & 34.3 & 36.3 & 38.8 & 32.9 & 37.3 & 35.4 & 35.2 \\
\hline & F2 & 42.4 & 36.5 & 41.8 & 40.2 & 33.1 & 45.0 & 34.4 & 37.5 \\
\hline & F3 & 46.3 & 34.2 & 38.2 & 39.6 & 28.4 & 30.6 & 27.1 & 28.7 \\
\hline \multicolumn{2}{|c|}{ F test and L.S.D' } & \multicolumn{2}{|c|}{ F test } & \multicolumn{2}{|c|}{ LSD $^{\prime}$} & \multicolumn{2}{|c|}{ F test } & \multicolumn{2}{|c|}{ LSD $^{\prime}$} \\
\hline \multicolumn{2}{|c|}{$\mathbf{M}$} & \multicolumn{2}{|c|}{ NS } & \multicolumn{2}{|c|}{------- } & \multicolumn{2}{|c|}{$*$} & \multicolumn{2}{|c|}{------- } \\
\hline \multicolumn{2}{|c|}{$\mathbf{G}$} & \multicolumn{2}{|c|}{$*$} & \multicolumn{2}{|c|}{1.37} & \multicolumn{2}{|c|}{ * } & \multicolumn{2}{|c|}{2.91} \\
\hline \multicolumn{2}{|c|}{$\mathbf{F}$} & \multicolumn{2}{|c|}{ NS } & \multicolumn{2}{|c|}{------- } & \multicolumn{2}{|c|}{ * } & \multicolumn{2}{|c|}{1.88} \\
\hline \multicolumn{2}{|c|}{$\mathbf{M} \times \mathbf{G}$} & \multicolumn{2}{|c|}{ NS } & & & & & & \\
\hline & & & & & & & & & \\
\hline & & & & & & & & & \\
\hline & $\times \mathbf{F}$ & & & & & & & & \\
\hline
\end{tabular}

Table 8. Effect of planting methods(M), genotypes(G), fertilization(F) and their interactions involved on oil content (\%)

\begin{tabular}{|c|c|c|c|c|c|c|c|c|c|}
\hline \multicolumn{2}{|c|}{ Seasons } & \multicolumn{3}{|c|}{2013} & \multirow{2}{*}{ Mean } & \multicolumn{3}{|c|}{2014} & \multirow{2}{*}{ Mean } \\
\hline $\begin{array}{c}\text { Planting } \\
\text { methods(M) }\end{array}$ & $\begin{array}{c}\text { Fertilization } \\
(\mathbf{F})\end{array}$ & G1 & G2 & G3 & & G1 & G2 & G3 & \\
\hline \multirow{3}{*}{ M1 } & F1 & 48.4 & 48.0 & 47.9 & 48.1 & 48.2 & 48.0 & 47.9 & 48.1 \\
\hline & F2 & 48.4 & 46.9 & 48.4 & 47.9 & 48.4 & 46.9 & 48.5 & 47.9 \\
\hline & F3 & 48.8 & 48.5 & 47.9 & 48.4 & 48.8 & 48.5 & 48.0 & 48.4 \\
\hline \multicolumn{2}{|c|}{ Mean } & 48.5 & 47.8 & 48.1 & 48.1 & 48.5 & 47.8 & 48.1 & 48.1 \\
\hline \multirow{3}{*}{ M2 } & F1 & 48.2 & 48.2 & 49.2 & 48.5 & 48.3 & 48.2 & 49.2 & 48.5 \\
\hline & F2 & 49.0 & 49.1 & 47.5 & 48.5 & 48.4 & 48.4 & 47.6 & 48.1 \\
\hline & F3 & 47.5 & 47.4 & 47.0 & 47.3 & 47.4 & 47.3 & 47.5 & 47.4 \\
\hline \multicolumn{2}{|c|}{ Mean } & 48.2 & 48.2 & 47.9 & 48.1 & 48.0 & 48.0 & 48.1 & 48.0 \\
\hline \multicolumn{2}{|c|}{ General mean } & 48.4 & 48.0 & 48.0 & 48.1 & 48.2 & 47.9 & 48.1 & 48.1 \\
\hline \multirow{3}{*}{$\mathbf{G} \times \mathbf{F}$} & F1 & 48.3 & 48.1 & 48.5 & 48.3 & 48.2 & 48.1 & 48.6 & 48.3 \\
\hline & F2 & 48.7 & 48.0 & 47.9 & 48.2 & 48.4 & 47.7 & 48.0 & 48.0 \\
\hline & F3 & 48.2 & 48.0 & 47.5 & 47.9 & 48.1 & 47.9 & 47.7 & 47.9 \\
\hline \multicolumn{2}{|c|}{ F test and L.S.D' } & \multicolumn{2}{|c|}{ F test } & \multicolumn{2}{|c|}{ LSD' } & \multicolumn{2}{|c|}{ F test } & \multicolumn{2}{|c|}{ LSD' $^{\prime}$} \\
\hline \multicolumn{2}{|c|}{$\mathbf{M}$} & \multicolumn{2}{|c|}{ NS } & \multicolumn{2}{|c|}{------ } & \multicolumn{2}{|c|}{$\mathrm{NS}$} & \multicolumn{2}{|c|}{-------- } \\
\hline \multicolumn{2}{|c|}{$\mathbf{G}$} & \multicolumn{2}{|c|}{$*$} & \multicolumn{2}{|c|}{0.33} & \multicolumn{2}{|c|}{ NS } & \multicolumn{2}{|c|}{----} \\
\hline \multicolumn{2}{|c|}{$\mathbf{F}$} & \multicolumn{2}{|c|}{$*$} & \multicolumn{2}{|c|}{0.28} & \multicolumn{2}{|c|}{ NS } & \multicolumn{2}{|c|}{----} \\
\hline \multicolumn{2}{|c|}{$\mathbf{M} \times \mathbf{G}$} & \multicolumn{2}{|c|}{$*$} & & & & & & \\
\hline & & & & & & & & & \\
\hline & & & & & & & S & & \\
\hline $\mathbf{M} \times$ & $\mathbf{S} \times \mathbf{F}$ & & & & & & & & \\
\hline
\end{tabular}


Table 9. Effect of planting methods(M), genotypes(G), fertilization(F) and their interactions involved on seed yield (kg fed.-1)

\begin{tabular}{|c|c|c|c|c|c|c|c|c|c|}
\hline \multicolumn{2}{|c|}{$\begin{array}{c}\text { Seasons } \\
\end{array}$} & \multicolumn{3}{|c|}{2013} & \multirow{2}{*}{ Mean } & \multicolumn{3}{|c|}{2014} & \multirow{2}{*}{ Mean } \\
\hline $\begin{array}{c}\text { Planting } \\
\text { methods(M) }\end{array}$ & $\begin{array}{c}\text { Fertilization } \\
(\mathbf{F})\end{array}$ & G1 & G2 & G3 & & G1 & G2 & G3 & \\
\hline \multirow{3}{*}{ M1 } & F1 & 467.7 & 496.7 & 458.1 & 474.2 & 515.4 & 555.5 & 698.9 & 589.9 \\
\hline & F2 & 511.7 & 366.4 & 513.3 & 463.8 & 814.8 & 638.2 & 818.0 & 757.0 \\
\hline & F3 & 456.2 & 405.8 & 397.0 & 419.7 & 654.9 & 514.6 & 525.6 & 565.0 \\
\hline \multicolumn{2}{|c|}{ Mean } & 478.5 & 423.0 & 456.1 & 452.5 & 661.7 & 569.4 & 680.8 & 637.3 \\
\hline \multirow{3}{*}{ M2 } & F1 & 598.6 & 392.4 & 389.2 & 460.1 & 811.4 & 639.4 & 777.2 & 742.7 \\
\hline & F2 & 640.2 & 504.9 & 489.0 & 544.7 & 413.7 & 601.0 & 323.1 & 445.9 \\
\hline & F3 & 507.1 & 507.1 & 488.8 & 501.0 & 545.2 & 590.2 & 652.1 & 595.8 \\
\hline \multicolumn{2}{|c|}{ Mean } & 582.0 & 468.1 & 455.7 & 501.6 & 590.1 & 610.2 & 584.1 & 594.8 \\
\hline \multicolumn{2}{|c|}{ General mean } & 530.3 & 445.6 & 455.9 & 477.2 & 625.9 & 589.8 & 632.5 & 616.0 \\
\hline \multirow{3}{*}{$\mathbf{G} \times \mathbf{F}$} & F1 & 533.1 & 444.6 & 423.7 & 467.1 & 663.4 & 597.4 & 738.0 & 666.3 \\
\hline & F2 & 576.0 & 435.6 & 501.1 & 504.2 & 614.2 & 619.6 & 570.5 & 601.4 \\
\hline & F3 & 481.7 & 456.5 & 442.9 & 460.4 & 600.0 & 552.4 & 588.9 & 580.4 \\
\hline \multicolumn{2}{|c|}{ F test and L.S.D' } & \multicolumn{2}{|c|}{ F test } & \multicolumn{2}{|c|}{ LSD $^{\prime}$} & \multicolumn{2}{|c|}{ F test } & \multicolumn{2}{|c|}{ LSD' $^{\prime}$} \\
\hline \multicolumn{2}{|c|}{$\mathbf{M}$} & \multicolumn{2}{|c|}{$*$} & \multicolumn{2}{|c|}{----- } & \multicolumn{2}{|c|}{$*$} & \multicolumn{2}{|c|}{------ } \\
\hline \multicolumn{2}{|c|}{$\mathbf{G}$} & \multicolumn{2}{|c|}{$*$} & \multicolumn{2}{|c|}{27.17} & \multicolumn{2}{|c|}{$*$} & \multicolumn{2}{|c|}{ n.s. } \\
\hline \multicolumn{2}{|c|}{$\mathbf{F}$} & \multicolumn{2}{|c|}{$*$} & \multicolumn{2}{|c|}{27.15} & \multicolumn{2}{|c|}{$*$} & \multicolumn{2}{|c|}{40.10} \\
\hline \multicolumn{2}{|c|}{$\mathbf{M} \times \mathbf{G}$} & \multicolumn{2}{|c|}{ * } & & & & & & \\
\hline & & & & & & & & & \\
\hline & & & & & & & & & \\
\hline $\mathbf{M} \times$ & $\times \mathbf{F}$ & & & & & & & & \\
\hline
\end{tabular}

Table 10. Effect of planting methods(M), genotypes(G), fertilization(F) and their interactions involved on oil yield (kg fed.-1)

\begin{tabular}{|c|c|c|c|c|c|c|c|c|c|}
\hline \multicolumn{2}{|c|}{ Seasons } & \multicolumn{3}{|c|}{2013} & \multirow{2}{*}{ Mean } & \multicolumn{3}{|c|}{2014} & \multirow[b]{2}{*}{ Mean } \\
\hline $\begin{array}{c}\text { Planting } \\
\text { methods(M) }\end{array}$ & $\begin{array}{c}\text { Fertilization } \\
\text { (F) }\end{array}$ & G1 & G2 & G3 & & G1 & G2 & G3 & \\
\hline \multirow{3}{*}{ M1 } & F1 & 226.4 & 238.4 & 219.4 & 228.1 & 248.4 & 266.6 & 334.8 & 283.7 \\
\hline & F2 & 247.7 & 171.8 & 248.4 & 222.2 & 394.4 & 299.3 & 396.7 & 362.6 \\
\hline & F3 & 222.6 & 196.8 & 190.2 & 203.1 & 319.6 & 249.6 & 252.3 & 273.5 \\
\hline \multicolumn{2}{|c|}{ Mean } & 232.1 & 202.2 & 219.4 & 217.6 & 320.9 & 272.2 & 327.5 & 306.5 \\
\hline \multirow{3}{*}{ M2 } & F1 & 288.5 & 189.1 & 191.5 & 223.1 & 391.9 & 308.2 & 382.4 & 360.2 \\
\hline & F2 & 313.7 & 247.9 & 232.3 & 264.2 & 200.2 & 290.9 & 153.8 & 214.5 \\
\hline & F3 & 240.9 & 240.4 & 229.7 & 237.0 & 258.4 & 279.2 & 309.7 & 282.4 \\
\hline \multicolumn{2}{|c|}{ Mean } & 280.5 & 225.6 & 218.3 & 241.3 & 283.2 & 292.9 & 280.9 & 285.5 \\
\hline \multicolumn{2}{|c|}{ General mean } & 256.7 & 213.9 & 218.8 & 229.5 & 301.7 & 282.5 & 304.2 & 296.3 \\
\hline \multirow{3}{*}{$\mathbf{G} \times \mathbf{F}$} & F1 & 257.5 & 213.8 & 205.5 & 225.6 & 319.7 & 287.3 & 358.7 & 321.8 \\
\hline & F2 & 280.5 & 209.1 & 240.0 & 243.0 & 297.3 & 295.5 & 273.8 & 288.7 \\
\hline & F3 & 232.2 & 219.1 & 210.4 & 220.5 & 288.6 & 264.6 & 280.9 & 278.0 \\
\hline \multicolumn{2}{|c|}{ F test and L.S.D' } & \multicolumn{2}{|c|}{ F test } & \multicolumn{2}{|c|}{ LSD $^{\prime}$} & \multicolumn{2}{|c|}{ F test } & \multicolumn{2}{|c|}{ LSD' $^{\prime}$} \\
\hline \multicolumn{2}{|c|}{$\mathbf{M}$} & \multicolumn{2}{|c|}{$*$} & \multicolumn{2}{|c|}{$-\cdots$} & \multicolumn{2}{|c|}{$*$} & \multicolumn{2}{|c|}{------ } \\
\hline \multicolumn{2}{|c|}{$\mathbf{G}$} & \multicolumn{2}{|c|}{$*$} & \multicolumn{2}{|c|}{12.88} & \multicolumn{2}{|c|}{ NS } & \multicolumn{2}{|c|}{---- } \\
\hline \multicolumn{2}{|c|}{$\mathbf{F}$} & \multicolumn{2}{|c|}{$*$} & \multicolumn{2}{|c|}{13.29} & \multicolumn{2}{|c|}{$*$} & \multicolumn{2}{|c|}{19.72} \\
\hline \multicolumn{2}{|c|}{$\mathbf{M} \times \mathbf{G}$} & \multicolumn{2}{|c|}{ * } & & & & & & \\
\hline & & & & & & & & & \\
\hline & & & & & & & & & \\
\hline & $\mathbf{3 \times F}$ & & & & & & & & \\
\hline
\end{tabular}


weight plant ${ }^{-1}$ could be explained on the basis that superiority of F2 fertilizer level with regard to number and weight of pods plant ${ }^{-1}$ or may be shelling percentage. Moreover, the most first order interactions involved in this respect had a significant effect on previous traits in both seasons. Also, the second order interaction (Planting methods $\times$ genotypes $\times$ fertilization levels) had a significant influence on mentioned traits in the two growing seasons except seed weight plant ${ }^{-1}$ in the second season. Thus, the highest mean values of seed index (102.7 and $88.5 \mathrm{~g})$ in the first and second seasons, respectively were obtained from terraces planting with sohag 110 genotype subjected to F3 fertilizer level in the first season and F1 in the second one. Furthermore, the highest seed weight plant ${ }^{-1}(49.9 \mathrm{~g})$ in the first season was obtained from terraces planting with Giza6 genotype subjected to F3 fertilizer level. This is logic since the same interaction produced the highest seed index and consequently seed weight plant ${ }^{-1}$.

\section{Seed oil content:}

Illustrated data in table 8 focuses that the planting methods didn't effected significantly on seed oil content (\%) in the two growing seasons. Here too, groundnut genotypes varied significantly in this respect. Thus, Giza 6 genotype surpassed the others tested genotypes and produced the highest mean values of seed oil content (48.4 and 48.2\%) in the first and second seasons, respectively. This is may be due to the genotypic behaviour in combination with the environmental conditions, which may be suitable for Giza 6 genotype than the rest genotypes. Moreover, the exhibited data reveal that fertilization had a significant effect on seed oil content in the first season only and the highest mean values of seed oil content (48.3 $\%)$ in both seasons was obtained from F1 $\left(20 \mathrm{~m}^{3}\right.$ cheep manure fed $\left.{ }^{-1}\right)$ while the lowest mean values of seed oil contents $(47.9 \%)$ in both seasons was produced when peanut plants fertilized by $100 \%$ of the recommended NPK fertilizers. The reduction in seed oil content by increasing nitrogen mineral fertilizer rate may be due to the role of nitrogen in transform metabolic products to amino acid then protein and reduced rate of fatty acid synthesis (Ali and Ahmed, 2012). Furthermore, the first order interaction had a significant effect on seed oil content in the first season, but in the second season most of it don't reached significant level. Also, the second order interaction had a significant effect on seed oil content in both seasons. Thus, the highest mean values of seed oil content $(49.2 \%)$ in both seasons was obtained from Sohag110 genotype cultivated by terraces method and subjected to $20 \mathrm{~m}^{3}$ cheep manure fertilization. Similar trend was obtained by Ahmed et al. (1997).

Seed and oil yields $\left(\mathrm{Kg} \mathrm{fed}^{-1}\right)$ :

Data illustrated in Tables 9 and 10 focuses that planting methods, genotypes, fertilization levels and their interactions involved affected sig- 
nificantly $(\mathrm{p} \leq 0.05)$ on seed yield and oil yields in both seasons, except the effect concerning genotypes in the second season which did not reached the significant level at 5\%. Thus, the maximum mean values of seed and oil yield in the first season (501.6 and $241.3 \mathrm{~kg}$ ) fed ${ }^{-1}$, respectively were obtained from M2 (terraces planting) while the furrow method gave the highest mean values in this respect in the second season( 637.0 and $306.5 \mathrm{~kg}$ $\mathrm{fed}^{-1}$ ) for seed and oil yields, respectively. Furthermore, the obtained data reveal that Giza 6 genotype produced the highest mean values of seed and oil yields (530.3 and $256.7 \mathrm{~kg} \mathrm{fed}^{-1}$ in the first season) while the superiority in the second season was to Sohage 110 genotype which gained the highest mean values of the mentioned traits (632.5 and $\left.304.2 \mathrm{~kg} \mathrm{fed}^{-1}\right)$ but, the differences between Sohage 110 and Giza 6 were not significant at 5\% level in the second season. This is to be expected since the same trend was obtained with regard to yield components and oil percentage. Similar findings were reported by Sarkees (2015). Moreover, the highest mean values of seed yield (504.2 and $\left.666.3 \mathrm{~kg} \mathrm{fed}^{-1}\right)$ in the first and second seasons, respectively and oil yield (243.0 and $\left.321.8 \mathrm{~kg} \mathrm{fed}^{-1}\right)$ in the first and second seasons, respectively were recorded from F2 and F1 fertilization treatment, respectively. The previous trend could be explained by the superiority in seed yield and oil percentage which controlled the oil yield trait. Here too, cultivated Giza 6 genotype by terraces method (M2) received F2 fertilization treatment gained the highest mean values $\left(640.2\right.$ and $\left.313.7 \mathrm{~kg} \mathrm{fed}^{-1}\right)$ of seed and oil yields, respectively in the first season, while in the second season the highest mean values (818.0 and $396.7 \mathrm{~kg} \mathrm{fed}^{-1}$ ) for seed and oil yields, respectively were obtained when Sohage 110 genotype was cultivated using furrow planting methods subjected to F2 fertilization treatment. The obtained results are in a good line with those obtained by Ahmed et al. (1997), Radwan and Awad (2002) and Ali \& Mahmoud (2012).

\section{References}

Abd El-Moez, M.R; A.L. Salah and A.H. Wanas (1999). Influence of some organic composts on yield, nutrients uptake and consumptive use of fennel and coriander plants and some soi physical properties. J. Agric. Sci. Mansoura Univ., 24(10): 6237-6253.

Ahmad, N. and M. Rahim (2007). Evaluation of promising groundnut,( Arachis hypogaea L). varieties for yield and other characters. J. Agric. Res. 45(3): 185-189.

Ahmed, M. K. A.; A. O. M. Saad ; A. T. Thalooth and M. O. Kabesh (1997). Fertilization of bio-fertilizers in yield crops production. 10-Yield response of groundnut to inorganic, organic and bio-fertilizers. Annals Agric. Sci., Ain Shams Univ., Cairo, 42(2): 365-375.

Ahmed, M.M.M. and E.B.A. Osman (2003). Response of peanut plants grown on a sandy calcareous soil to fertilization with farmyard manure and organo-mineral fertilizer prepared from some sugar can wastes. Assiut J. Agric. Sci., 34 (6): 337347. 
Ali, E.A. and S.Y. Ahmed (2012). Influence of nitrogen rates and foliar spray by different concentration of copper on sesame seed and oil yields as well as nitrogen use efficiency in sandy soil. Res. J. Agric. \& Biol. Sci., 8(2): 174-178.

Ali, E.A. and A.M. Mahmoud (2012). Effect of combination between organic and mineral fertilization on productivity of some safflower genotypes. World Journal of Agricultural Sciences 8 (2): 134-140.

A.O.A.C. (1995). Association of Official Analytical Chemists. Official methods of analysis, $16^{\text {th }}$ Ed. AOAC International, Washington, D.C., USA.

FAO (2014): Faostat,fao.org/download/Q/QC/E.

Gomez, K.A. and A.A. Gomez (1984). Statistical Procedures For Agriculture Research. A Wiley - Inter Science Publication, John wiley sons, lnc. New York, USA.

Mahrous,, N. M. ; S. A. Safina; H. H. Abo Taleb and S. M. El-Behlak.(2015). Integrated use of organic, inorganic and bio fertilizers on yield and quality of two peanut (Arachis hypogaea L.) cultivars grown in a sandy saline soil. American-Eurasian J. Agric. \& Environ. Sci., 15 (6): 1067-1074.

Meena, R.S, R.S; Yadav and V.S. Meena (2014). Response of groundnut (Arachis hypogaea L.) varieties to sowing dates and NP fertilizers under western dry zone of india. Bangladesh J. Bot. 43(2): 169-173.

Michigan State University (1983). MSTAT-C Micro-Computer Statistical Programe, Version2. Michigan State University, East Lansing, USE.

Orlando, J.L.; S.F. Filho and A.A. Rodella (1991). Effects of filter cake applications on sugar yield in Brazil. Sugar J., 54(3): 22-24.

Radwan, S.M.A. and N. M. Awad (2002). Effect of soil amendment with various organic wastes with multi-biofertilizer on yield of peanut plants in sandy soil. J. Agric. Sci., Mansoura Univ., 27(5): 3129-3138.

Sarkees N.A. (2015). Effect of Sowing Dates on Development, Seed Yield and Quality of Some Peanut (Arachis hypogaea L.) Genotypes. Jordan J. Agric. Sci., 11(2) $: 67-380$.

Sikora, L.J. and M.J. Azard (1993). Effect of compost fertilizer combinations on wheat yields. Compost Science and Utilization, 2: 93-96.

Singh, G.; O.P. Sing R.S. Singh R.A. Yadav and B. Singh (1991). Effect of press mud and fertilizer application on yield and quality of cane in flood affected condition. Bharatiya Sugar, 27: 27-30.

Sun, R.L.; B.Q. Zhao and L.S. Zhu (2003). Effects of long-term fertilization on soil enzyme activities and its role in adjusting-controlling soil fertility. J. Plant Nutrition and Fertilizer Science 9, 406-410. 
تقييم بعض التراكيب الوراثية من الفول السوداني تحت طريقتين للزراعة ومستويات مختلفة من التسميا

ابراهيم عبد الباقي رزق الفار'، السعدي عبد الحميد علي'، وحيد عبد العزيز الصاوي`و وعلي حسان

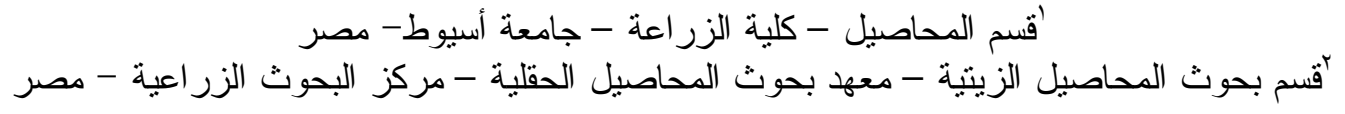

الملخص

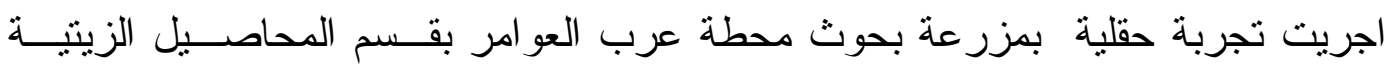

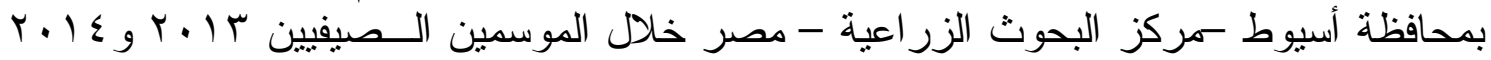

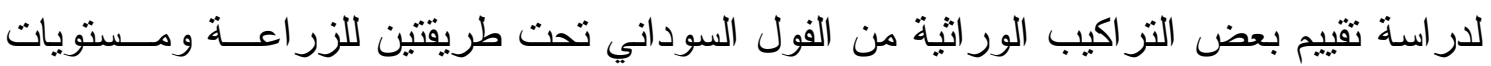

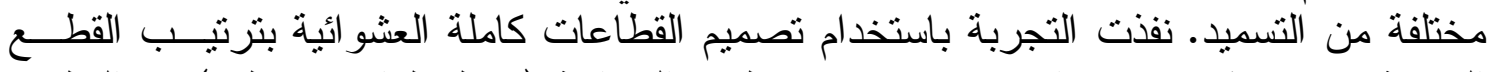

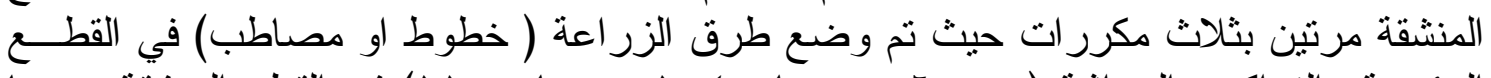

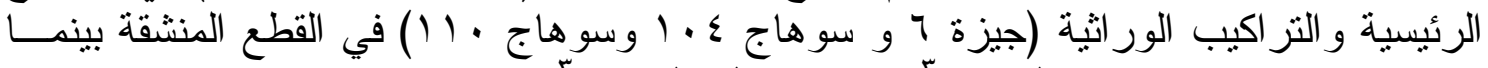

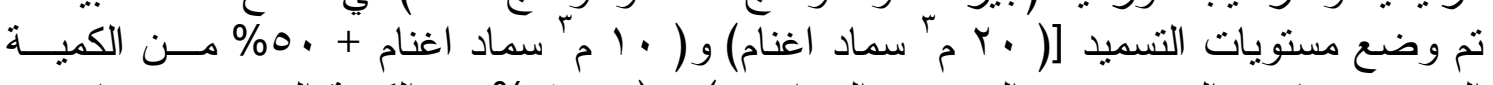

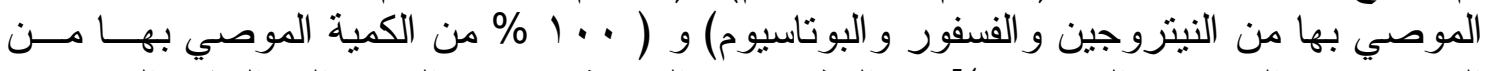

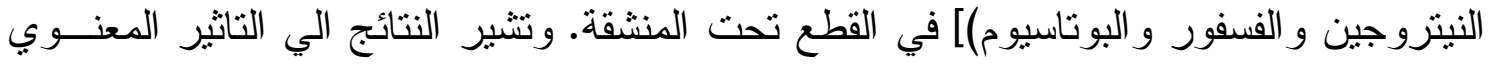

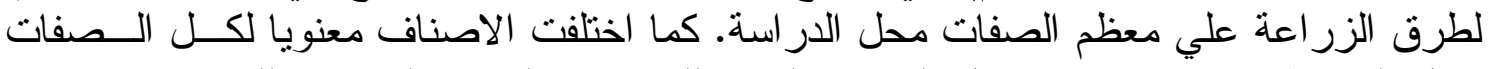

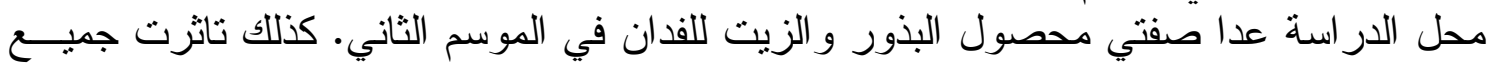

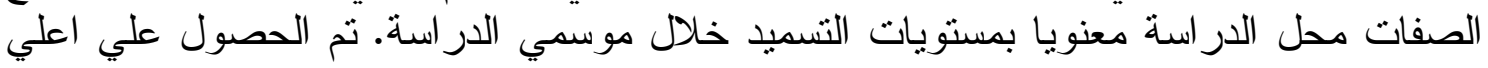

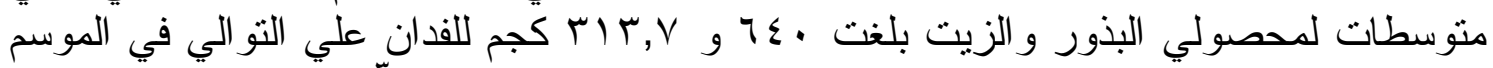

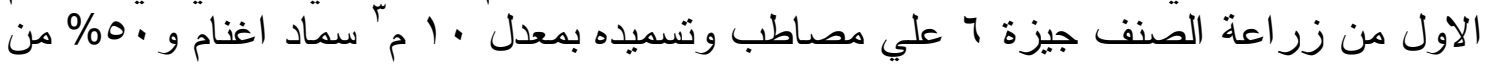

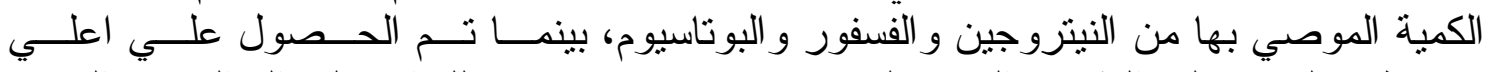

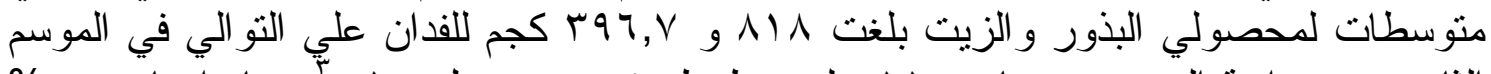

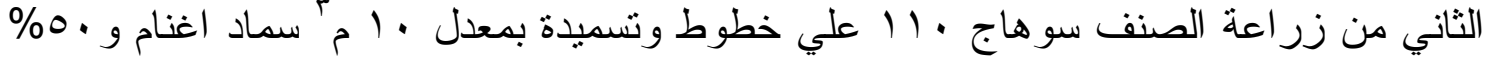

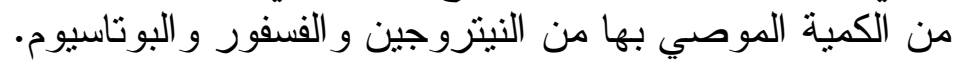

